DOI: $10.3901 / J M E .2020 .03 .130$

\title{
摩擦纳米发电机表面织构的优化设计
}

\author{
杨潍旭 $^{1}$ 王晓力 $^{2}$ 陈 平 ${ }^{1}$ \\ (1. 北京科技大学机械工程学院 北京 100083 ; \\ 2. 北京理工大学机械与车辆学院 北京 100081)
}

\begin{abstract}
摘要: 摩擦纳米发电机是基于自驱动纳米技术并以接触/摩擦起电和静电感应为基础的微/纳机电动力系统, 提高其起电性能 的有效手段之一是将微/纳织构制备在接触界面上。采用理论与试验相结合的方法开展摩擦纳米发电机表面织构的优化设计研 究。建立摩擦纳米发电机织构界面黏附接触模型, 探讨外载荷、织构面密度及宽度对摩擦纳米发电机起电性能的影响规律, 获得不同外载荷下使摩擦纳米发电机起电性能最优的织构参数, 并制备织构化摩擦纳米发电机, 构建起电性能测试平台, 试 验验证摩擦纳米发电机最优织构的设计效果。结果表明: 不同外载荷下使摩擦纳米发电机起电性能达到最优的织构参数不同; 相比于织构宽度, 织构面密度对于摩擦纳米发电机起电性能的影响更为显著; 所构建的理论模型结果与试验结果具有较好的 一致性, 可为摩擦纳米发电机表面织构的设计提供理论指导。
\end{abstract}

关键词: 摩擦纳米发电机; 表面织构; 接触力学; 接触起电

中图分类号: TH117

\section{Optimal Design of Surface Texture in Triboelectric Nanogenerators}

\section{YANG Weixu ${ }^{1}$ WANG Xiaoli ${ }^{2}$ CHEN Ping ${ }^{1}$}

(1. School of Mechanical Engineering, University of Science and Technology Beijing, Beijing 100083;

2. Department of Mechanical Engineering, Beijing Institute of Technology, Beijing 100081)

\begin{abstract}
Triboelectric nanogenerator (TENG) is a micro/nano electromechanical power system which is based on the ideas of self-powered nanotechnology and the mechanisms of triboelectric and electrostatic induction. An effective way to improve power generation of TENGs is to introduce micro-/nano-textures onto the contact surface. The optimum design of surface texture in TENGs is studied by combining simulation with experiment. An adhesive contact model for TENG with textured interface is established to discuss the influence of applied load, texture density and width on the power generation of TENG and obtain optimal texture parameters for maximum TENG power generation under different applied loads. In addition, TENGs with textured surfaces are fabricated and the test platform of electrical output for TENGs is constructed to verify the design effect of the optimal texture of TENG. It is shown that the optimal texture parameters of TENGs under different applied loads vary. Compared with the texture width, the influence of texture density on the power generation of TENG is more significant. In addition, the simulation results are in good agreement with the experimental results, which indicates that the theoretical model can provide the guideline for the texture design in TENG.
\end{abstract}

Key words: triboelectric nanogenerator; surface texture; contact mechanics; contact electrification

\section{0 前言}

摩擦纳米发电机(Triboelectric nanogenerator, TENG)是基于自驱动纳米技术并以接触/摩擦起电和 静电感应为基础的微/纳机电动力系统 ${ }^{[1]}$ 。法向接触分离式 TENG 结构简单, 可靠性高, 应用领域广泛,

* 中国博士后基金(2019M660449)、国家自然科学基金(51735001)和国家重 点研发计划(2018YFC0810500)资助项目。20190727 收到初稿, 20191216 收到修改稿
因此引起研究者的广泛关注 ${ }^{[2]}$ 。接触起电的电荷量与 接触副表面电荷密度及有效接触面积密切相关, 因此 提高摩擦纳米发电机起电性能的一个重要手段是通过 将具有微/纳特征尺度的织构制备在接触界面上来增 加有效接触面积 ${ }^{[3-4]}$ 。例如, FAN 等 ${ }^{[3]}$ 在聚合物表面制 备均匀规则的微米级织构(线型、立方体、棱雉), 发现 相比于光滑无织构表面, 表面具有微米级织构的 TENG 的输出开路电压与短路电流均有显著的提高。 然而, 目前表面织构参数的确定多采用经验与试探的 手段, 摩擦纳米发电机表面织构的优化设计仍是难题。 
接触力学的核心是建立外载荷与接触面积的关 系, 因此接触力学与接触起电密切相关, 可以作为 摩擦纳米发电机表面织构设计的理论基础。对于表 面具有微/纳织构的摩擦纳米发电机而言, 接触区域 小到微/纳米尺寸, 经典接触理论不再适用, 需要考 虑两表面间的黏附力 (例如范德华力和静电力)建立 黏附接触力学模型 ${ }^{[5-6]}$ 。目前黏附接触力学模型主要 包括解析模型和数值模型。解析模型如 $\mathrm{JKR}^{[7]}$ 、 $\mathrm{DMT}^{[8]} 、 \mathrm{MD}^{[9]}$ 主要针对球体与光滑平面间或两光滑 球体间的黏附接触, 将两物体间的黏附作用简化为 其相邻表面之间的黏附作用, 因而不适用于分析织 构化摩擦纳米发电机中光滑平面与微/纳织构表面 的黏附接触。数值模型如基于 Lifshitz-Hamaker 方 法 $^{[10-11]}$ 的模型通过对物体间所有分子间的相互作用 进行累加求和来获得两物体间的黏附力, 因此可以 被扩展应用于摩擦纳米发电机织构界面黏附接触的 分析。然而, 目前相关的表面织构的优化设计理论 与试验研究尚少, 难以为工程中改善摩擦纳米发电 机起电性能的织构优化设计提供依据。

本文采用理论与试验相结合的方法开展摩擦纳 米发电机表面织构的优化设计研究。建立摩擦纳米 发电机织构界面黏附接触模型并数值求解, 探讨外 载荷、织构面密度及宽度对摩擦纳米发电机起电性 能的影响规律, 获得不同外载荷下使摩擦纳米发电 机起电性能最优的织构参数, 并制备织构化摩擦纳 米发电机, 搭建起电测试平台, 试验验证摩擦纳米 发电机最优织构的设计效果。

\section{1 黏附接触力学模型}

图 $1 \mathrm{a}$ 和 $1 \mathrm{~b}$ 分别为法向接触-分离式摩擦纳米发 电机的接触与分离状态, 其中 $V_{\mathrm{oc}}$ 代表接触电极与 背部电极之间的开路电压, $d$ 为接触电极与聚合物

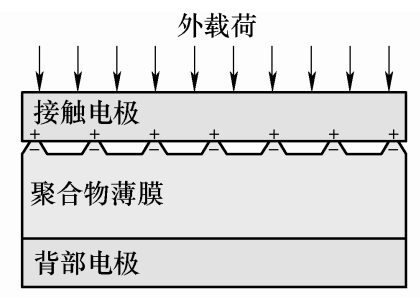

(a) 接触状态

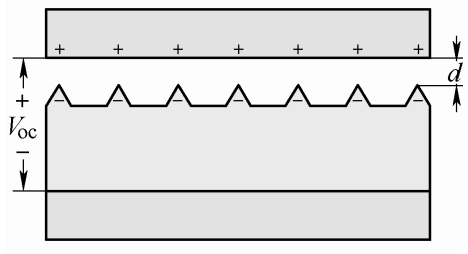

(b) 分离状态

图 1 法向接触-分离式摩擦纳米发电
薄膜之间的距离。由于表面织构均匀分布, 为简便 起见, 取单一织构单元与电极的接触作为分析对象, 如图 $2 \mathrm{a}$ 和 $2 \mathrm{~b}$ 所示。

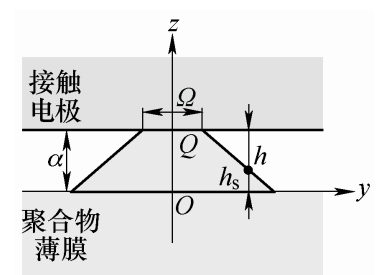

(a) 接触状态

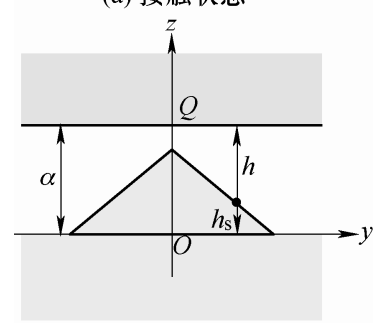

(b) 分离状态

图 2 接触电极与聚合物薄膜单一织构单元黏附接触模型

由于金属接触电极与聚合物薄膜的弹性模量相 差较大, 因此黏附接触计算时将接触电极视为刚体, 而织构化聚合物薄膜视为弹性体。接触电极与织构 聚合物薄膜的间距 $h(x, y)$ 可以表示为

$$
h(x, y)=-\alpha-h_{\mathrm{s}}(x, y)+u(x, y)
$$

式中, $\alpha$ 为接触电极底部与 $z=0$ 平面之间的距离, 称为接近距离。当接触电极位于 $z=0$ 平面之上时, 定义 $\alpha<0$, 否则 $\alpha>0$ 。 $h_{\mathrm{s}}(x, y)$ 为织构高度分布, 对 于常应用于 TENG 的四棱雉织构, 如图 3 所示, $h_{\mathrm{s}}(x, y)$ 可以表示为

$$
h_{\mathrm{s}}(x, y)=\left\{\begin{array}{l}
b-\frac{b(|x+y|+|x-y|)}{a} \\
0 \text { 其他 }
\end{array}|x|,|y| \leqslant \frac{a}{2}\right.
$$

式中, $a 、 b$ 分别代表棱雉织构的宽度及高度, $c$ 为 织构单元宽度, 则织构面密度为 $S_{\mathrm{p}}=a^{2} / c^{2}$ 。

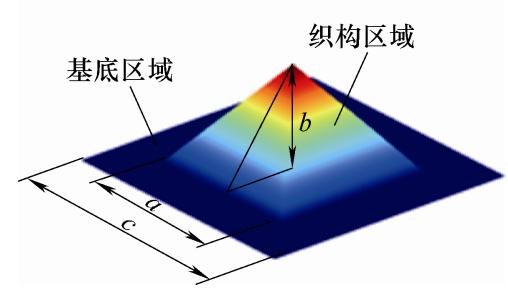

图 3 单一棱雉织构单元示意图

式(1)中 $u(x, y)$ 代表织构表面的弹性变形量, 根 据 Boussinesq 公式 ${ }^{[12]}$ 可表示为

$$
u(x, y)=\frac{1}{\pi E^{*}} \iint \frac{p(\xi, \eta) \mathbf{d} \xi \mathbf{d} \eta}{\sqrt{(x-\xi)^{2}+(y-\eta)^{2}}}
$$

式中, $E^{*}=E /\left(1-v^{2}\right), E$ 和 $v$ 分别代表聚合物薄膜的弹 
性模量和泊松比。 $p(x, y)$ 为接触表面之间的黏附接触 应力, 可以表示为

$$
p(x, y)=\left\{\begin{array}{l}
p_{\mathrm{vdW}}(x, y)+p_{\text {rep }}(x, y)+p_{\text {ele }}(x, y)(x, y) \in \Omega \\
p_{\mathrm{vdW}}(x, y)+p_{\text {rep }}(x, y) \text { 其他 }
\end{array}\right.
$$

式中, $p_{\mathrm{vdW}}(x, y)$ 为范德华吸引作用力, 起源于材料 中分子的固有极化; $p_{\text {rep }}(x, y)$ 为排斥接触作用力, 起 源于分子间距较小时电子云的相互重叠, 基于 Lifshitz-Hamaker 方法 ${ }^{[10-11]}$, 接触电极与聚合物薄膜 之间的范德华吸引力和排斥接触力可以通过对描述 间两非键合分子之间相互作用势的 Lennard-Jones 势函数进行两步体积分获得，可以表示为

$$
\begin{gathered}
p_{\mathrm{vdW}}(x, y)=-\frac{A_{\mathrm{H}}}{6 \pi h^{3}(x, y)} \\
p_{\text {rep }}(x, y)=\frac{A_{\mathrm{H}} l_{0}^{6}}{90 \pi h^{9}(x, y)}
\end{gathered}
$$

式中, $A_{\mathrm{H}}$ 为 Hamaker 常数; $l_{0}$ 为物体间平衡间距。 $p_{\text {ele }}(x, y)$ 为静电吸引作用力, 起源于具有不同得失电 能力的接触电极与聚合物薄膜之间电荷转移而产生 的接触电荷。根据平行板电容器模型, 可以表示为 ${ }^{[13]}$

$$
p_{\text {ele }}(x, y)=-\frac{\sigma(x, y)^{2}}{2 \varepsilon_{3}}\left(\frac{B_{1}}{B_{1}+h(x, y)}\right)^{2}
$$

式中, $\sigma(x, y)$ 为聚合物薄膜的表面电荷密度; $\varepsilon 3$ 为接 触电极及聚合物薄膜所处介质的介电常数; $B 1=T 1 / K 1, T 1$ 和 $K 1$ 分别为聚合物薄膜的厚度和相对 介电常数。若忽略电荷的耗散作用, 静电作用仅存 在于接触或已经发生过接触的区域。本文将接触应 力为压应力的区域定义为接触区域 $\Omega$, 接触区域内 棱雉织构表面的表面积为参与接触起电的有效接触 面积 $S_{\mathrm{c}}{ }^{[14]}$ 。则接触电极与织构化聚合物薄膜之间的 总黏附相互作用力为

$$
F=\iint p(x, y) \mathbf{d} x \mathbf{d} y
$$

在求解过程中，保持织构化聚合物薄膜不动， 使接触电极从一初始间距逐步接近织构化聚合物薄 膜, 即逐步减小接近距离 $\alpha$, 计算每一 $\alpha$ 值下的弹 性变形 $u(x, y)$ 、黏附接触应力 $p(x, y)$ 、总黏附相互作 用力 $F$ 及接触区域 $\Omega$, 直到总黏附作用力 $F$ 达到设 定的施加载荷值 $F_{\mathrm{app}} / n_{\mathrm{u}}$, 其中 $F_{\mathrm{app}}$ 代表 TENG 器件 的外载荷, $n_{\mathrm{u}}$ 为 $\mathrm{TENG}$ 内织构单元个数, 若假设 TENG 器件尺寸为 $S_{\mathrm{d}}$, 则 $n_{\mathrm{u}}=S_{\mathrm{d}} / c^{2}$ 。根据接触区域计 算此施加载荷 $F_{\text {app }}$ 下参与接触起电的有效接触面积 $S_{\mathrm{c}}$ 以及输出开路电压 $V_{\mathrm{oc}}=\sigma S_{\mathrm{c}} d / \varepsilon_{0}(a+c)^{2}$, 其中, $\varepsilon_{0}$ 为 真空的介电常数。计算流程如图 4 所示, 计算中主 要采用非精确牛顿法、二次共轭梯度稳定算法以及
FFT 快速算法 ${ }^{[15]}$ 。

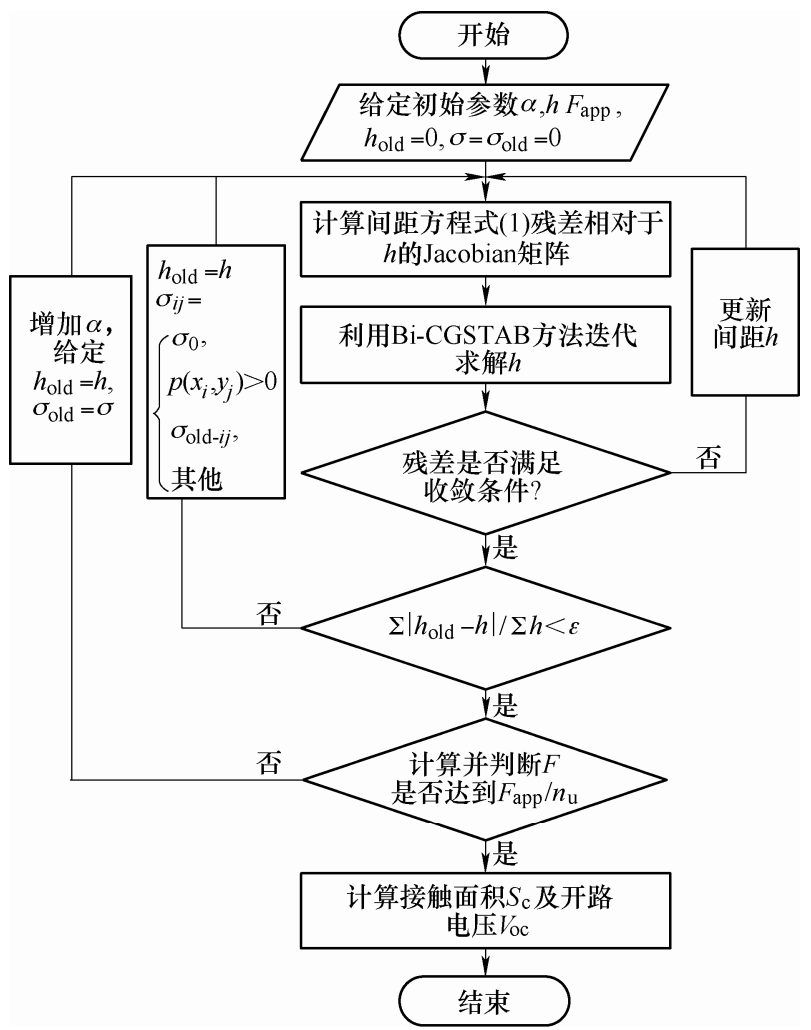

图 4 电极与聚合物薄膜黏附接触计算流程图

\section{2 理论计算结果与讨论}

利用理论模型，探讨外载荷、织构面密度及宽 度对于 TENG 有效接触面积及开路电压的影响规 律，获得不同外载荷下使 TENG 起电性能最优的织 构参数。计算中，接触电极及聚合物薄膜材料分别 为铜 $(\mathrm{Cu})$ 和聚二甲基硅氧烷(PDMS), 织构选择为规 则分布的四棱雉阵列，相关计算参数如下：等效弹 性模量 $E^{*}=1.5 \mathrm{MPa}$, Hamaker 常数 $A_{\mathrm{H}}=3.34 \times 10^{-13} \mathrm{~J}$, 平衡间距 $l_{0}=0.46 \mu \mathrm{m}$, 表面电荷密度 $\sigma=2.78 \mu \mathrm{C} / \mathrm{m}^{2}$, 器件尺寸 $S_{\mathrm{d}}=1 \mathrm{~cm} \times 1 \mathrm{~cm}$ 。

\section{1 外载荷的影响}

本节探究外载荷对棱雉织构 TENG 有效接触面 积及输出开路电压的影响, 织构尺寸参数为: 织构 面密度 $S_{\mathrm{p}}=45 \%$, 织构宽度 $a=10 \mu \mathrm{m}$, 织构高度 $b=0.7 a^{[16]}$ 。有效接触面积随外载荷的变化如图 5 所 示, 可以发现, 随着外载荷的增加, 有效接触面积 先显著增加, 后缓慢增加。当外载荷较小时 $\left(F_{\mathrm{app}}<100\right.$ $\mathrm{N})$, 棱雉织构表面与金属电极尚未完全接触，随着 外载荷的增加, 接触区域显著增加, 如图 5 中 $20 \mathrm{~N}$ 、 $40 \mathrm{~N}$ 及 $100 \mathrm{~N}$ 外载荷下棱雉织构表面的接触应力分 布图所示，其中深色区域代表接触应力为负的区域， 即非接触区域，因此此时有效接触面积随外载荷的 
增加而显著增加; 当外载荷较大时 $\left(F_{\text {app }} \geqslant 100 \mathrm{~N}\right)$, 棱 锥织构表面基本完全与金属电极发生接触, 如图 5 中 $100 \mathrm{~N}$ 及 $120 \mathrm{~N}$ 外载荷下棱雉织构表面的接触应力 分布图所示, 其中深色区域代表非接触区域, 因此 此时有效接触面积随外载荷的增加而缓慢增加。

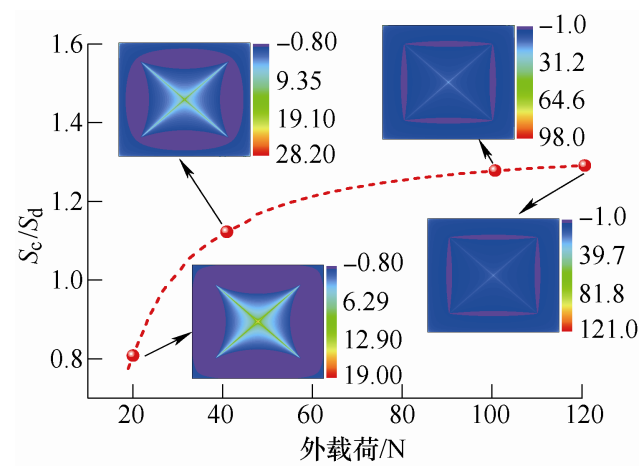

图 5 有效接触面积随外载荷的变化

图 6 为摩擦纳米发电机的输出开路电压随外载 荷的变化, 可以发现, 随着外载荷的增加, 开路电 压呈现出与有效接触面积相同的规律, 即先显著增 加, 后缓慢增加。

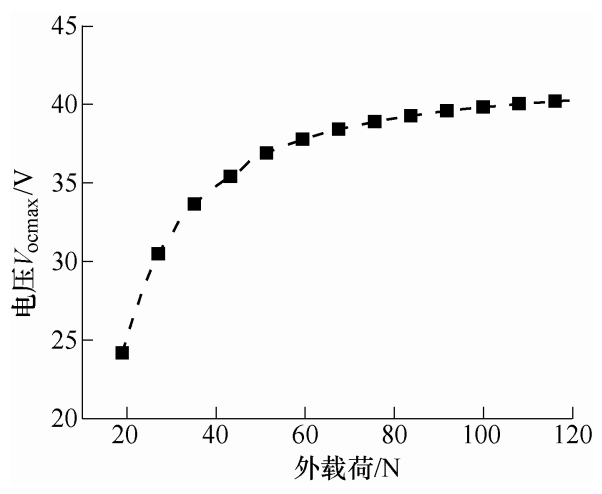

图 6 开路电压随外载荷的变化

\section{2 织构面密度的影响}

本节探究较小外载荷 $20 \mathrm{~N}$ 以及较大外载荷 120 $\mathrm{N}$ 作用下织构面密度对棱雉织构 TENG 有效接触面 积及输出开路电压的影响, 织构尺寸参数为: 织构 面密度 $S_{\mathrm{p}}=25 \% \sim 45 \%$, 织构宽度 $a=10 \mu \mathrm{m}$, 织构高 度 $b=0.7 a^{[16]}$ 。图 7 为 $20 \mathrm{~N}$ 外载荷时 TENG 有效接 触面积随织构面密度的变化, 可以发现此时有效接 触面积随面密度的增加而降低, 这是由于棱雉织构 表面尚未完全与金属电极接触, 随着面密度的增加, 与金属电极接触的基底区域减少, 因此有效接触面 积随面密度的增加而降低, 如图 7 中 $20 \mathrm{~N}$ 外载荷下 面密度分别为 $25 \%$ 、35\%以及 $45 \%$ 的棱雉织构表面 的接触应力分布图所示, 其中紫色区域代表接触应 力为负的区域, 即非接触区域。

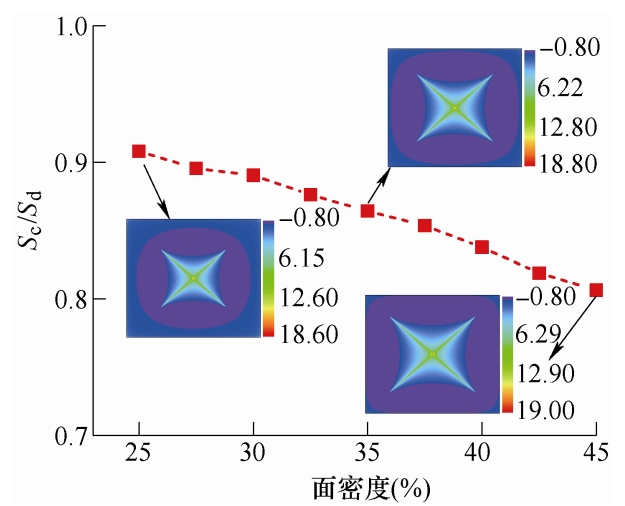

图 $720 \mathrm{~N}$ 外载荷时有效接触面积随织构面密度的变化

图 8 为 $120 \mathrm{~N}$ 外载荷时 TENG 有效接触面积随 织构面密度的变化。可以发现, 与 $20 \mathrm{~N}$ 外载荷时情 况不同, $120 \mathrm{~N}$ 外载荷时有效接触面积随面密度的 增加而增加, 此时棱雉织构表面基本完全与金属电 极发生接触, 如图 8 中 $120 \mathrm{~N}$ 外载荷时不同面密度 棱雉织构表面的接触应力分布图所示, 其中紫色区 域代表非接触区域, 由于面密度大的棱雉织构表面 具有更大的表面积, 因此 $120 \mathrm{~N}$ 外载荷时有效接触 面积呈现出如此趋势。

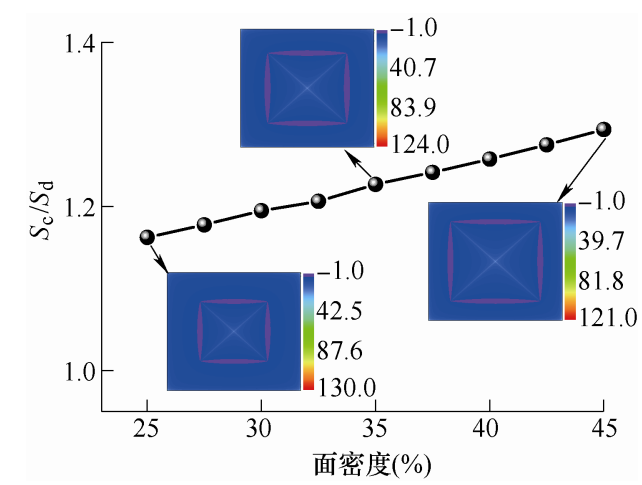

图 $8120 \mathrm{~N}$ 外载荷时有效接触面积随织构面密度的变化

图 9 为 $20 \mathrm{~N}$ 及 $120 \mathrm{~N}$ 外载荷时棱雉织构面密度 对于 TENG 最大开路电压的影响。随着面密度的变 化, 棱雉织构 TENG 开路电压呈现出与有效接触面积 相同的规律。当外载荷较小时, 开路电压随面密度的 增加而减小，在本研究范围内使开路电压达到最大的 最优织构面密度为 $25 \%$; 而外载荷较大时, 开路电压 随面密度的增加而增加, 最优织构面密度为 $45 \%$ 。

\section{3 织构宽度的影响}

本节探究 $20 \mathrm{~N}$ 及 $120 \mathrm{~N}$ 外载荷下织构宽度对棱 雉织构 TENG 有效接触面积及输出开路电压的影 响, 织构尺寸参数为: 织构面密度 $S_{\mathrm{p}}=25 \%$, 织构宽 度 $a=5 \sim 10 \mu \mathrm{m}$, 织构高度 $b=0.7 a^{[16]}$ 。图 10 为 $20 \mathrm{~N}$ 外载荷时 TENG 有效接触面积随织构宽度的变化, 可以发现此时有效接触面积随织构宽度的增加而略 有降低, 这是由于棱雉织构表面尚未完全与金属电 


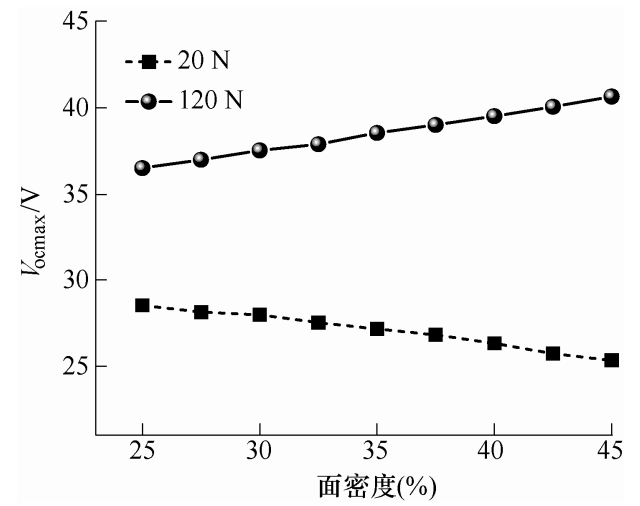

图 9 开路电压随织构面密度的变化

极接触, 随着织构宽度的增加, 与金属电极接触的 基底区域略有减少，如图 10 中 $20 \mathrm{~N}$ 外载荷下织构 宽度分别为 $5.0 \mu \mathrm{m} 、 7.5 \mu \mathrm{m}$ 以及 $10.0 \mu \mathrm{m}$ 的棱雉织 构表面的接触应力分布图所示, 其中紫色区域代表 非接触区域, 因此有效接触面积随织构宽度的增加 而略有降低。

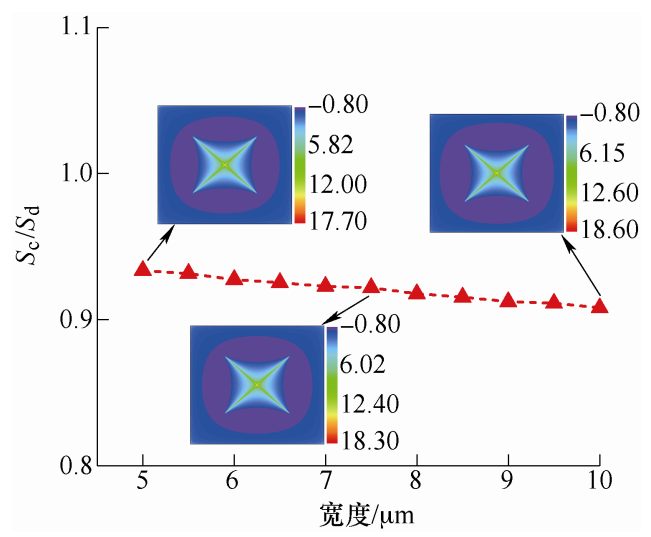

图 $1020 \mathrm{~N}$ 外载荷时有效接触面积随织构宽度的变化

图 11 为 $120 \mathrm{~N}$ 外载荷时 TENG 有效接触面积 随织构宽度的变化。可以发现, $120 \mathrm{~N}$ 外载荷时有 效接触面积基本不随织构宽度的增加而变化, 这是 由于此时棱雉织构表面基本完全与金属电极发生接 触, 如图 11 中 $120 \mathrm{~N}$ 外载荷时不同宽度棱雉织构表 面的接触应力分布图所示，其中紫色区域代表非接 触区域, 由于面密度相同时不同宽度的织构表面积 均相同, 因此有效接触面积不随宽度的变化而改变。

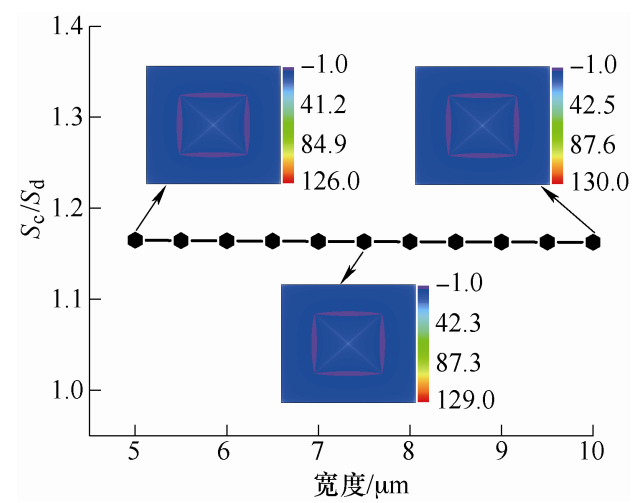

图 $11120 \mathrm{~N}$ 外载荷时有效接触面积随织构宽度的变化
图 12 为 $20 \mathrm{~N}$ 及 $120 \mathrm{~N}$ 外载荷时棱雉织构宽度对 于 TENG 最大开路电压的影响。随着宽度的变化, 棱 雉织构 TENG 开路电压呈现出与有效接触面积相同 的规律。当外载荷较小时, 开路电压随宽度的增加而 略有减小, 在本研究范围内使开路电压达到最大的最 优织构宽度为 $5 \mu \mathrm{m}$; 而外载荷较大时, 开路电压随宽 度的增加而基本不变。并且, 相比于织构宽度, 织构 面密度对于 TENG 开路电压的影响更为显著。

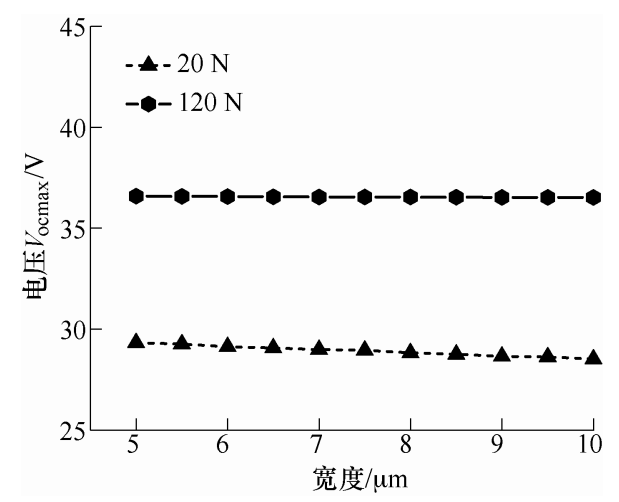

图 12 开路电压随织构宽度的变化

\section{3 试验方法}

\section{1 制备}

制备织构化 PDMS 薄膜主要包括硅模板的制备 及复型两大步骤。其中，硅模板为具有指定宽度、高 度及面密度的倒棱雉凹坑阵列图形的母模板, 主要采 用热氧化、光学光刻以及氢氧化钾湿法刻蚀等工艺进 行制备; 而复型则通过旋涂及揭膜等工艺加工出与硅 模板图案相反, 即具有棱雉凸起阵列的 PDMS 薄膜。 棱雉织构 PDMS 薄膜加工尺寸如表 1 所示。图 13a 和 $13 b$ 分别为样品 $\mathrm{B}$ 和 $\mathrm{C}$ 的棱雉织构 SEM 图。

表 1 薄膜尺寸参数

\begin{tabular}{cccc}
\hline 织构样品 & $\mathrm{A}$ & $\mathrm{B}$ & $\mathrm{C}$ \\
\hline 宽度 $/ \mu \mathrm{m}$ & 5 & 10 & 10 \\
面密度 $(\%)$ & 25 & 25 & 45 \\
\hline
\end{tabular}

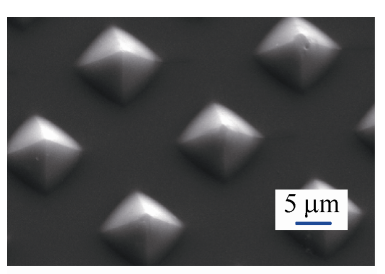

(a)

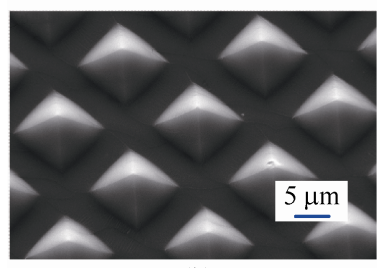

(b)

图 13 棱雉织构 SEM 图 


\section{2 起电性能测试}

摩擦纳米发电机起电性能测试平台如图 14 所 示, 主要包括运动控制系统和起电性能测量系统, 其中, 运动控制系统主要包括线性马达、压力传感 器、嵌入式控制器以及可编程逻辑控制器(PLC)程序 等, 为 TENG 提供可控外载荷的循环接触分离运动; 起电性能测量系统主要包括静电计及计算机, 测量 记录 TENG 接触分离过程中产生的电学信号。

起电性能测试步骤如下: (1) 将接触电极固定于 线性马达滑杆连接件, 贴有背部电极的织构化 PDMS 薄膜固定于压力传感器; (2) 在 PLC 程序中 设定外载荷参数, 并通过嵌入式控制器传递至线性 马达; (3) 启动线性马达, 即可实现 TENG 的循环 接触分离运动。运动过程中, 将接触电极及背部电 极分别与静电计相连, 即可测量记录 TENG 输出开 路电压。

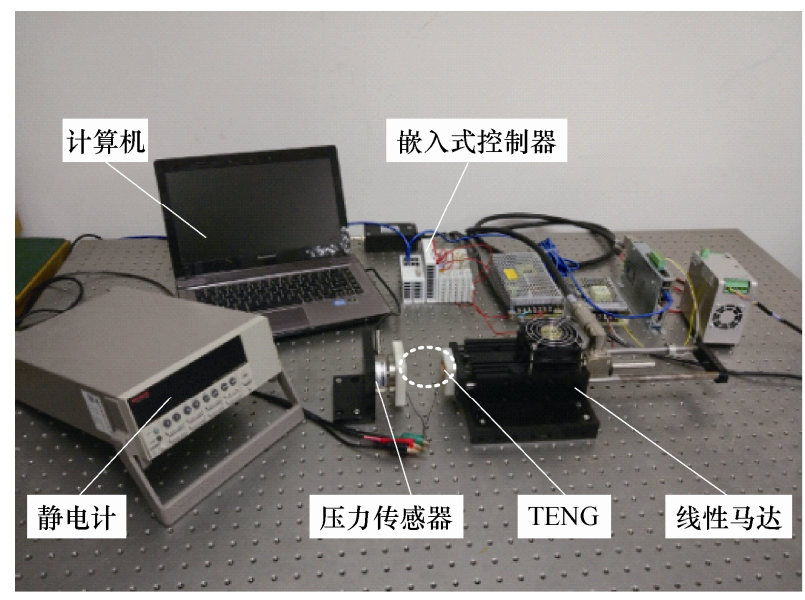

图 14 TENG 起电性能测试平台

\section{4 试验结果与讨论}

利用起电性能测试平台, 测量 $20 \mathrm{~N}$ 及 $120 \mathrm{~N}$ 外 载荷下不同棱雉织构 TENG 的输出开路电压, 为了 对比, 同时测试光滑表面 TENG(编号为 $\mathrm{O}$ )的输出开 路电压。试验中 $\mathrm{TENG}$ 器件尺寸为 $1 \mathrm{~cm} \times 1 \mathrm{~cm}$, 温度 $25 \sim 30^{\circ} \mathrm{C}$, 相对湿度 $25 \% \sim 50 \%$ 。

开路电压测试结果如图 15 所示, 可以发现, 当 外载荷较小时, 本试验范围内棱雉织构 TENG 输出 的最高开路电压为 $28.1 \mathrm{~V}$, 相比于光滑表面 TENG 提升了 $28.3 \%$, 最优织构面密度为 $25 \%$, 最优织构 宽度为 $5 \mu \mathrm{m}$; 当外载荷较大时, 棱雉织构 TENG 输 出的最高开路电压为 $40.9 \mathrm{~V}$, 相比于光滑表面 TENG 提升了 $25.5 \%$, 最优织构面密度为 $45 \%$, 最 优织构参数的试验结果与理论计算结果一致。

图 16 为样品编号为 $\mathrm{C}$ 的棱雉织构摩擦纳米发 电机在外载荷 $20 \mathrm{~N}$ 下持续运行 $1500 \mathrm{~s}$ 所输出的开
路电压结果。可以看出, 织构化 TENG 在 $0 \mathrm{~s}$ 时刻 输出开路电压约为 $23.1 \mathrm{~V}$, 在 $1500 \mathrm{~s}$ 时刻输出开路 电压约为 $23.2 \mathrm{~V}$, 即, 在循环运行使用后, 仍可以 保持稳定的电输出, 说明本文所制备的具有棱雉织 构的摩擦纳米发电机具有良好的输出稳定性。

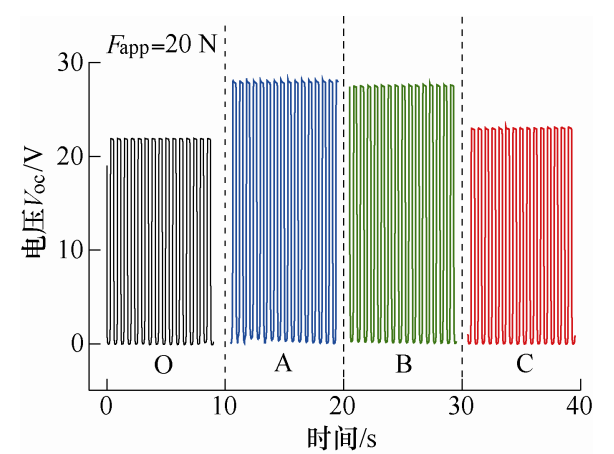

(a)

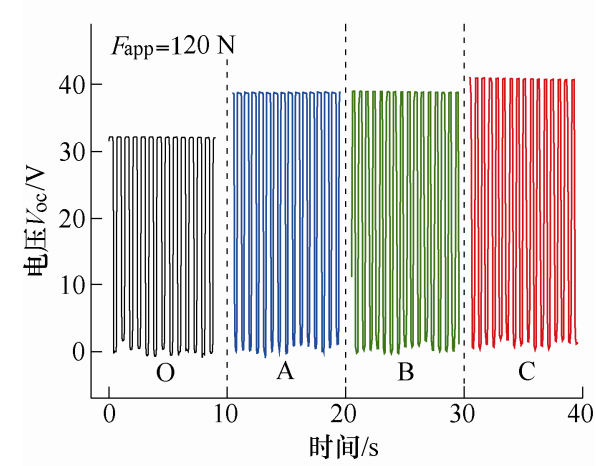

(b)

图 15 不同 TENG 样品的输出开路电压

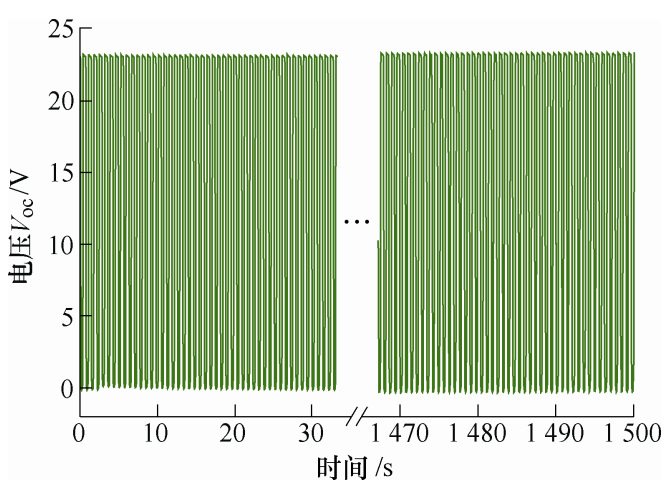

图 $16 \mathrm{TENG}$ 开路电压随时间的变化

\section{5 结论}

(1) 摩擦纳米发电机有效接触面积及开路电压 随外载荷的增加呈现先显著增加, 后缓慢增加的趋 势; 外载荷较小时, 有效接触面积及开路电压随织 构面密度及宽度的增加而减小; 外载荷较大时, 有 效接触面积及开路电压随织构面密度的增加而增 加, 随织构宽度的增加而基本不变。

(2) 在本文研究范围内, 不同外载荷下使开路 电压达到最优的织构参数不同, 当外载荷较小时, 
最优织构面密度和宽度分别为 $25 \%$ 和 $5 \mu \mathrm{m}$; 当载荷 较大时，最优织构面密度 $45 \%$ 。

（3）相比于织构宽度, 织构面密度对于摩擦纳 米发电机起电性能的影响更为显著。

（4）本文所构建理论模型的计算结果与试验结 果具有较好的一致性, 可为摩擦纳米发电机表面织 构的设计提供理论参考。

\section{参 考 文 献}

[1] WANG Z L, CHEN J, LIN L. Progress in triboelectric nanogenerators as a new energy technology and self-powered sensors[J]. Energy \& Environmental Science, 2015，8(8): 2250-2282.

[2] WANG H, SHI M, ZHU K, et al. High performance triboelectric nanogenerators with aligned carbon nanotubes[J]. Nanoscale, 2016, 8(43): 18489-18494.

[3] FAN F-R, LIN L, ZHU G, et al. Transparent triboelectric nanogenerators and self-powered pressure sensors based on micropatterned plastic films[J]. Nano Letters, 2012, 12(6): 3109-3114.

[4] TCHO I W, KIM W G, JEON S B, et al. Surface structural analysis of a friction layer for a triboelectric nanogenerator[J]. Nano Energy，2017，42: 34-42.

[5] 韩振华, 石万凯, 肖洋轶, 等. 基于 JKR 模型的粗糙 圆柱表面线黏着接触分析[J]. 机械工程学报, 2016, 52(19): 116-122.

HAN Zhenhua, SHI Wankai, XIAO Yangyi, et al. Adhesive contact analysis between rough surfaces based on JKR model[J]. Journal of Mechanical Engineering, 2016, 52(19): 116-122.

[6] YANG W, WANG X, LI H, et al. Comprehensive contact analysis for vertical-contact-mode triboelectric nanogen erators with micro-/nano-textured surfaces[J]. Nano Energy, 2018, 51: 241-249.

[7] JOHNSON K L, KENDALL K, ROBERTS A D. Surface energy and the contact of elastic solids[J]. Proceedings of the Royal Society of London A: Mathematical and Physical Sciences, 1971, 324(1558): 301-313.

[8] DERJAGuin B V, MULLER V M, TOPROV Y P J. Effect of contact deformation on the adhesion of particles[J]. Journal of Colloid and Interface Science, 1975, 53(2): 314- 326.

[9] MAUGIS D. Adhesion of spheres: The JKR-DMT transition using a Dugdale model[J]. Journal of Colloid and Interface Science, 1992，150(1): 243-269.

[10] LIFSHITZ E. The theory of molecular attractive forces between solids[J]. Soviet Physics, 1956, 2: 73-83.

[11] ISRAELACHVILI J N. Intermolecular and surface forces [M]. Pittsburgh: Amercian Academic Press, 2011.

[12] JOHNSON K L, JOHNSON K L. Contact mechanics[M]. Cambridge: Cambridge University Press, 1987.

[13] HORN R G, SMITH D T. Contact electrification and adhesion between dissimilar materials[J]. Science, 1992, 256(5055): 362-364.

[14] YANG W, WANG X, LI H, et al. Fundamental research on the effective contact area of micro-/nano-textured surface in triboelectric nanogenerator[J]. Nano Energy, 2019, 57: 41-47.

[15] ZHANG Y, WANG X, LI H, et al. Adhesive behavior of micro/nano-textured surfaces[J]. Applied Surface Science, 2015, 329: 174-183.

[16] JIN C, KAI D S, JONES M, et al. On the contact behavior of micro-/nano-structured interface used in vertical-contact-mode triboelectric nanogenerators[J]. Nano Energy, 2016, 27: 68-77.

作者简介: 杨滩旭(通信作者), 女, 1992 年出生, 博士, 特聘副教授。 主要研究方向为 MEMS 黏附接触力学以及接触起电基础。

E-mail: karenyang1992@163.com

王晓力, 女, 1965 年出生, 博士, 教授, 博士研究生导师。主要研究方 向为纳米摩擦学、黏附接触力学以及微纳制造。

E-mail: xiaoli_wang@bit.edu.cn

陈平, 女, 1973 年出生, 博士, 教授, 博士研究生导师。主要研究方向 为机械设计及理论、摩擦磨损与系统工程。

E-mail: chenp@ustb.edu.cn 